\title{
Engineering integrin signaling for promoting embryonic stem cell self-renewal in a precisely defined niche
}

\author{
Seung Tae Lee ${ }^{\mathrm{a}}$, Jung Im Yun ${ }^{\mathrm{a}}$, Yun Suk Jo ${ }^{\mathrm{a}}$, Mayumi Mochizuki ${ }^{\mathrm{a}}$, André J. van der Vlies ${ }^{\mathrm{a}}$, \\ Stephan Kontos ${ }^{\mathrm{a}}$, Jong Eun Ihm ${ }^{\mathrm{a}}$, Jeong M. Lim ${ }^{\mathrm{c}}$, Jeffrey A. Hubbell ${ }^{\mathrm{a}, \mathrm{b}, *}$ \\ a Institute of Bioengineering, Ecole Polytechnique Fédérale de Lausanne, CH-1015 Lausanne, Switzerland \\ ${ }^{\mathrm{b}}$ Institute of Chemical Sciences and Engineering, Ecole Polytechnique Fédérale de Lausanne, CH-1015 Lausanne, Switzerland \\ ${ }^{\mathrm{c}}$ Major in Biomodulation, WCU, Department of Agricultural Biotechnology, Seoul National University, 151-921 Seoul, Republic of Korea
}

\section{A R T I C L E I N F O}

\section{Article history:}

Received 27 August 2009

Accepted 25 October 2009

Available online 18 November 2009

\section{Keywords:}

Adhesion molecule

Cell signaling

ECM (extracellular matrix)

Hydrogel

Stem cell

\begin{abstract}
A B S T R A C T
We present development and use of a 3D synthetic extracellular matrix (ECM) analog with integrinspecific adhesion ligands to characterize the microenvironmental influences in embryonic stem cell (ESC) self-renewal. Transcriptional analysis of 24 integrin subunits followed by confirmation at the translational and functional levels suggested that integrins $\alpha_{5} \beta_{1}, \alpha_{v} \beta_{5}, \alpha_{6} \beta_{1}$ and $\alpha_{9} \beta_{1}$ play important roles in maintenance of stemness in undifferentiated mouse ESCs. Using the well-defined matrix as a tool to activate integrins $\alpha_{5} \beta_{1}$ plus $\alpha_{v} \beta_{5}, \alpha_{6} \beta_{1}$ and $\alpha_{9} \beta_{1}$, individually and in combination, differential integrin activation was demonstrated to exert exquisite control over ESC fate decisions. Simultaneous ligation of these four integrin heterodimers promoted self-renewal, as evidence by prolonged SSEA-1, Oct 4 and Nanog expression, and induced Akt1 kinase signaling along with translational regulation of other stemness-related genes. The biofunctional network we have designed based on this knowledge may be useful as a defined niche for regulating ESC pluripotency through selective cell-matrix interactions, and the method we present may be more generally useful for probing matrix interactions in stem cell selfrenewal and differentiation.
\end{abstract}

(c) 2009 Elsevier Ltd. All rights reserved.

\section{Introduction}

The nature of the niche that enables prolonged self-renewal of ESCs remains only partially elucidated. An often used, but poorly understood, such environment is provided by mouse embryonic fibroblasts (MEFs), which present substantial difficulties in systematic investigations because of their presentation of myriad signals to the ESCs. Several attempts have been described to replace MEF feeder layers with ECM proteins and matrices such as Matrigel, ECM derived from MEFs, etc. [1-3]. These biologically derived materials are also very complex and are not subject to control at the molecular level. Synthetic hydrogel and fibrillar matrices have been explored, in an attempt to replace such complicated biological materials with defined artificial materials for short-term ESC culture [4,5], yet these materials still do not present the fullness of the ECMs repertoire of molecular characteristics. Sophisticated photochemical degradation approaches have been developed to explore the dynamic nature of cell-material interactions in the

\footnotetext{
* Corresponding author. EPFL, SV/IBI/LMRP, Station 15, CH-1015 Lausanne, Switzerland. Tel.: +4121693 9682; fax: +41216939665.

E-mail address: jeffrey.hubbell@epfl.ch (J.A. Hubbell).
}

niche in artificial systems, for example the factors controlling stem cell chondrogenic differentiation [6].

ECM components can induce a variety of signals for functional regulation of cells, and the integrin family of cell surface adhesion receptors consisting of $\alpha$ and $\beta$ subunits [7] regulates numerous such interactions related to biological process that include cell attachment, spreading, proliferation, survival, morphogenesis and gene expression [8-11], as well as critical steps of differentiation in early embryogenesis [7,12-16]. However, despite considering ECM signaling as an important factor in ESC self-renewal fate decisions $[17,18]$, the literature remains unclear about the function of integrins in maintenance of the ESC self-renewal, except for self-renewal of adult stem cell [19-21], in part due to a lack of well characterized research tools. We have developed a synthetic ECM analog based on branched poly(ethylene glycol) (PEG) crosslinked with matrix metalloproteinase (MMP)-sensitive peptides and functionalized with peptide adhesion ligand [22,23]. Cells are able to interact with the matrix via the coupled adhesion peptides and to remodel the matrix via the MMPs they activate and present at the cell surface. Here, we functionalize these materials with multiple integrinactivating ligands with good control over the amounts and identities of the adhesion ligands for the purpose of elucidating the 
influence exerted by the ECM on ESCs in their self-renewal fate decisions. These methods may more generally provide a defined niche for investigation of matrix interactions in stem cell selfrenewal and differentiation.

\section{Materials and methods}

\subsection{ESC culture}

The mouse ESCs E14tg2a (CRL-1821, ATCC, Manassas, VA) were routinely cultured on a feeder layer of MEFs treated with $10 \mu \mathrm{g} / \mathrm{ml}$ mitomycin C (SigmaAldrich, St. Louis, MO) in $5 \% \mathrm{CO}_{2}$ at $37^{\circ} \mathrm{C}$ in Dulbecco's modified Eagle's medium (DMEM; Gibco Invitrogen, Grand Island, NY) supplemented with $15 \%$ (v/v) heatinactivated fetal bovine serum (FBS; HyClone, Logan, UT), $0.1 \mathrm{~mm} \beta$-mercaptoethanol (Gibco Invitrogen), 1\% (v/v) nonessential amino acids (NEAA; Gibco Invitrogen), $1 \mathrm{~mm}$ sodium pyruvate (Sigma-Aldrich), 2 mm L-glutamine (Gibco Invitrogen), a 1\% $(\mathrm{v} / \mathrm{v})$ lyophilized mixture of penicillin and streptomycin (Gibco Invitrogen) and 1000 units/ml mouse leukemia inhibitory factor (LIF) (Chemicon International, Temecula, CA) (herein referred to as ESC culture medium). Subpassage was performed every third day and the medium was changed daily during subculture.

\subsection{Quantitative real-time $P C R$}

All the specific primer sequences shown in Supplementary Table 1 were designed with cDNA sequences obtained from GenBank for mouse and by Primer3 software (Whitehead Institute/MIT Center for Genome Research). Extraction of total mRNA from each group was conducted by using a RNeasy Plus Mini Kit (Qiagen, Valencia, CA) and cDNA was synthesized from $\sim 1 \mu \mathrm{g}$ of total mRNA using a Reverse Transcription System (Promega, Madison, WI). Gene expression was estimated by real-time quantitative PCR using iQ ${ }^{\mathrm{TM}} \mathrm{SYBR}^{\circledR}$ Green Supermix (Bio-Rad Laboratories, Hercules, CA). The PCR amplification was performed on the Bio-Rad iCycler iQ system (Bio-Rad Laboratories) in a final volume of $25 \mu \mathrm{l}$ using cycling parameters ( 3 min, $95^{\circ} \mathrm{C} ; 30 \mathrm{~s}, 95^{\circ} \mathrm{C} ; 30 \mathrm{~s}, 60^{\circ} \mathrm{C} ; 30 \mathrm{~s} 72{ }^{\circ} \mathrm{C}$, with the latter three steps repeated for 40 times). The melting curve data were collected to check the PCR specificity. By identifying the absence of dimerization between primers through control real-time $\mathrm{PCR}$ reaction lacking template, $300 \mathrm{~nm}$ in the all primers was selected as the final optimized concentration. The mRNA level of each sample for each gene was normalized to that of $\beta$-actin mRNA. Relative mRNA level was presented as $2^{-\Delta \Delta \mathrm{ct}}$ where $\mathrm{Ct}=$ threshold cycle for target amplification, $\Delta \mathrm{Ct}=\mathrm{Ct}_{\text {target gene }}$ (specific genes for each sample) $-\mathrm{Ct}_{\text {internal reference }}\left(\beta\right.$-actin for each sample), and $\Delta \Delta \mathrm{Ct}=\Delta \mathrm{Ct}_{\text {sample }}$ (treatment sample in each experiment) $-\Delta \mathrm{Ct}_{\text {calibrator }}$ (control sample in each experiment).

\subsection{Western blot}

Cell lysates were prepared by treatment of M-PER ${ }^{\circledR}$ Mammalian Protein Extraction Reagent (Pierce, Woburn, MA) supplemented with protease inhibitor cocktail (Roche Diagnostics, Indianapolis, IN) with protein concentration determination with a NanoDrop ${ }^{\mathrm{TM}} 1000$ spectrophotometer (Thermo Fisher Scientific, Rockford, IL). Subsequently, the cell lysates ( 160 or $240 \mu \mathrm{g}$ ) were fractionated by 7.5 , 10 or $15 \%(\mathrm{v} / \mathrm{v})$ SDS-polyacrylamide gel, according to the molecular size of the protein of interest, and transferred onto a polyvinylidene difluoride membrane (Millipore, Billerica, MA). After blocking with $5 \%(\mathrm{w} / \mathrm{v})$ skimmed milk (Bio-Rad laboratories) overnight at $4{ }^{\circ} \mathrm{C}$, the membrane was washed three times with PBST consisting of PBS (pH 7.4; Gibco Invitrogen) and 0.1\% Tween-20 (Sigma-Aldrich), and incubated with the primary antibodies listed in the Supplementary Table 2. After washing three times with PBST, the primary antibodies were detected by the appropriate horseradish peroxidase (HRP)-conjugated secondary antibodies listed in the Supplementary Table 2 and developed with enhanced chemiluminescent substrate (Pierce). Protein bands on membranes were visualized with CL-XPosur$\mathrm{e}^{\mathrm{TM}}$ Film (Thermo Fisher Scientific) and the intensities of individual protein bands were quantified by densitometry using a Bio-Rad Imaging System Quantity One ver. 4.5.1 with ChemiDoc XRS (Bio-Rad Laboratories).

\subsection{Formation of PEG-based hydrogel, insertion of ESCs into PEG-hydrogel and} culture

The PEG-based hydrogel was formed by the conjugation of vinylsulfone (SigmaAldrich)-functionalized 3arm-PEG (PEG-VS) synthesized as described [24] and dicysteine-containing peptides with an intervening matrix metalloproteinase (MMP)-specific cleavage site (Ac-GCRD-GPQGIWGQ-DRCG-NH $\mathrm{N}_{2}$ ) (herein referred to as crosslinker) through a Michael-type addition reaction [25]. For making a typical $10 \%$ (w/v) PEG-hydrogel into which ESC were incorporated, all the precursor solutions of PEG-VS (dissolved in ESC culture medium), crosslinker (dissolved in pH 8.0 $0.3 \mathrm{~m}$ HEPES buffer) and ESCs (suspended in ESC culture medium) were mixed. For incorporation of ESCs into the PEG-hydrogel with specific integrin-binding adhesion peptides, firstly, the PEG-VS precursor solution was reacted with the precursor solution containing the adhesion peptides (in $\mathrm{pH} 8.00 .3 \mathrm{~m}$ HEPES buffer) for $30 \mathrm{~min}$ at room temperature and this solution was then mixed with the precursor solutions of crosslinker and ESCs. Subsequently, gelation of a circular disk derived from $6 \mu \mathrm{l}$ of the mixed precursor solutions occurred within $30 \mathrm{~min}$ at $37^{\circ} \mathrm{C}$ [24] and the ESCs encapsulated into PEG-hydrogel were cultured in a humidified atmosphere of $5 \% \mathrm{CO}_{2}$ in air at $37{ }^{\circ} \mathrm{C}$ in the ESC culture medium and the medium was exchanged every day.

\subsection{Peptide synthesis and purification}

Peptides synthesis on solid resin (Novasyn TGR, Novabiochem, Laeufelfingen, Switzerland) was performed using an automated peptide synthesizer (Chemspeed, Augst, Switzerland) with standard Fmoc/HBTU/HOBT chemistry [24,25]. Purification of synthesized peptides was conducted by reverse phase chromatography on a C18 column (Biocad 700E; PerSpective Biosystems). The sequence of purified peptides were confirmed by matrix-assisted laser desorption ionization/time-of-fligh (MALDI-TOF) mass spectrometry. The details of all the peptides utilized are shown in the Supplementary Table 3.

\subsection{Flow cytometric analysis}

The ESC population fixed in $4 \%(\mathrm{v} / \mathrm{v})$ paraformaldehyde (Sigma-Aldrich) was washed in ice cold PBS and $1 \times 10^{6}$ ESC from the ESC population were distributed separately for analysis of stage specific embryonic antigen (SSEA)-1, Oct4 and Nanog expression. For staining SSEA-1, cell surface protein, the distributed ESCs were incubated for $1 \mathrm{~h}$ at $4{ }^{\circ} \mathrm{C}$ with primary anti-SSEA-1 (as shown in Supplementary Table 2) diluted in $2 \%(\mathrm{v} / \mathrm{v}$ ) FBS-containing PBS, and this primary antibody was detected by incubating fluorescently conjugated secondary antibody (as shown in Supplementary Table 2 ) for $1 \mathrm{~h}$ at $4{ }^{\circ} \mathrm{C}$. For staining Oct 4 and Nanog, which exist in the nucleus as a transcription factor, ESCs were incubated for $1 \mathrm{~h}$ at $4{ }^{\circ} \mathrm{C}$ with fluorescently conjugated anti-Oct4 and anti-Nanog (as shown in Supplementary Table 2) diluted in Hanks' Balanced Salt Solution (HBSS; Gibco Invitrogen) supplemented with $0.1 \%(w / v)$ saponin (AppliChem Gmbh, Darmstadt, Germany) and 0.05\% (w/v) sodium azide (Sigma-Aldrich). Subsequently, after washing sufficiently, the stained cells resuspended in $500 \mu \mathrm{l}$ PBS were analyzed using flow cytometry, on a CyAn ${ }^{\mathrm{TM}}$ ADP Analyzer (Beckman Coulter, Inc., Fullerton, CA), and FLOWJO Ver. 7.2.5 software program (Tree Star, Inc., Ashland, OR).

\subsection{ELISA}

Preparation of cell lysates was conducted by using methods recommended from each ELISA manufacturer and the amount of cell lysates were quantified with a NanoDrop ${ }^{\mathrm{TM}} 1000$ spectrophotometer. Subsequently, analysis by ELISA of total Stat3 (Gibco Invitrogen), phospho-Tyr705-Stat3 (Gibco Invitrogen), total $\beta$-catenin (Cell Signaling, Beverly, MA), total Akt1 (Cell Signaling), and phospho-Ser 473-Akt1 (Cell Signaling) protein assays were performed according to manufacturer's instructions.

\subsection{Statistical analysis}

All the numerical data derived from each experiment were analyzed statistically by the Statistical Analysis System (SAS) program. Moreover, when a significance of the main effects was detected by analysis of variance (ANOVA) in the SAS package, the least-square or DUNCAN methods were conducted to compare among treatments. Significant differences among treatments were determined where the $p$ value was less than 0.05 .

\section{Results}

\subsection{Identification of integrin heterodimers expressed in undifferentiated embryonic stem cells (ESCS)}

To provide guidance regarding which integrins may be present in the undifferentiated state, we monitored expression of integrin heterodimers in undifferentiated mouse ESCs. Transcriptional levels of genes encoding total 24 integrin subunits (Fig. 1a,d) were quantified, and integrin $\alpha_{5}, \alpha_{6}, \alpha_{8}, \alpha_{9}$ and $\alpha_{v}$ and integrin $\beta_{1}, \beta_{3}, \beta_{4}, \beta_{5}$ and $\beta_{7}$ showed higher expression levels than other subunits. By contrast, integrin $\alpha_{1}, \alpha_{3}, \alpha_{4}, \alpha_{7}, \alpha_{10}, \alpha_{11}, \alpha_{\mathrm{L}}$ and $\alpha_{N}$ were minimally transcribed, while no expression of integrin $\alpha_{2}, \alpha_{D}$ and $\alpha_{x}$ and integrin $\beta_{2}, \beta_{6}$ and $\beta_{8}$ were observed. When translation of the integrin $\alpha$ subunits showing increased transcription $\left(\alpha_{5}, \alpha_{6}, \alpha_{9}\right.$ and $\alpha_{v}$ ) was evaluated, integrin $\alpha_{5}$ subunit showed the highest translation in the amount of glycosylated mature and non-glycosylated immature proteins (Fig. 1b,f,h). The total amounts of integrin $\alpha_{6}$ and 
a

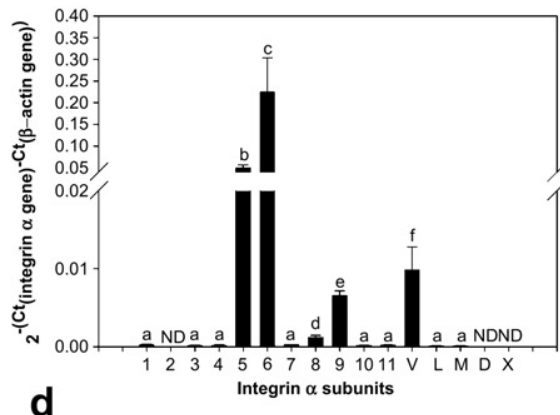

d
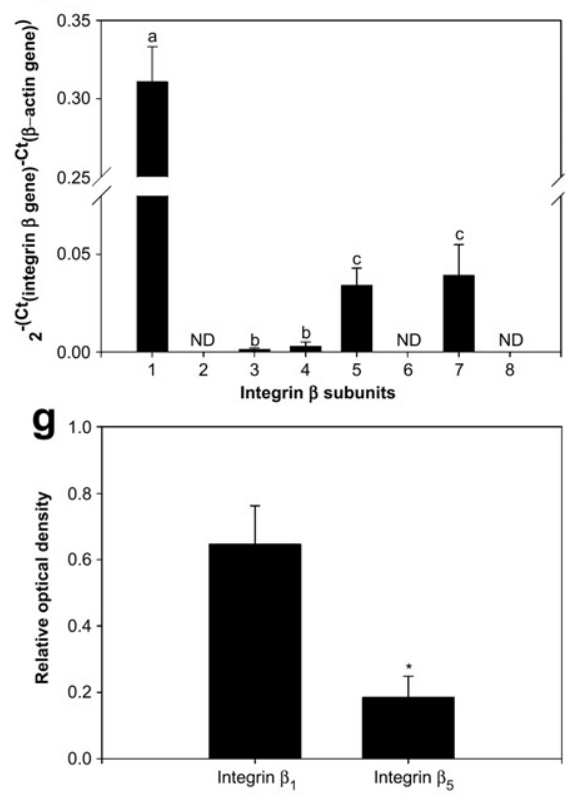

b

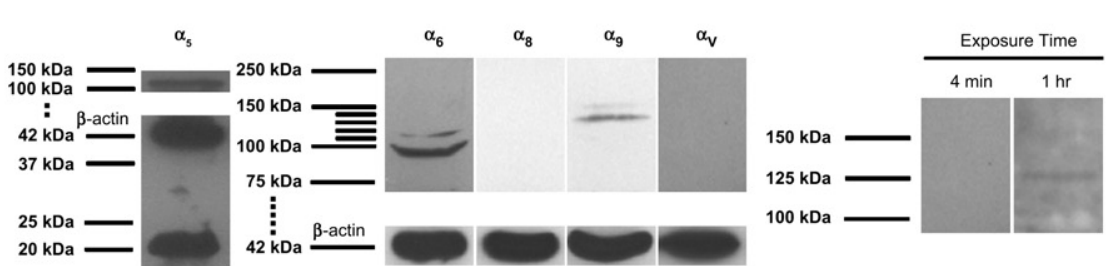

e

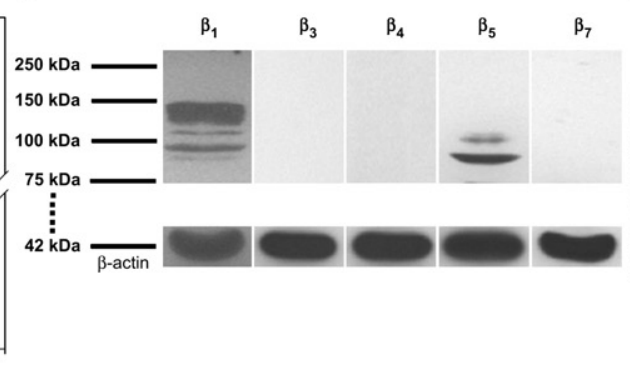

h

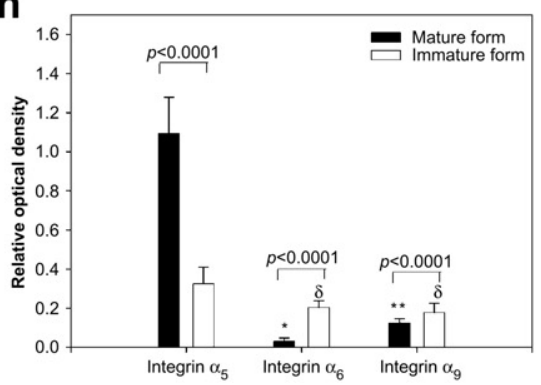

C

f

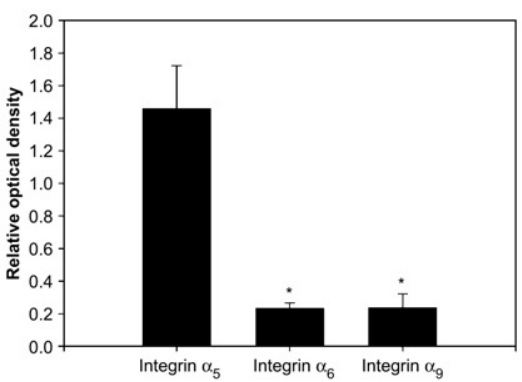

i

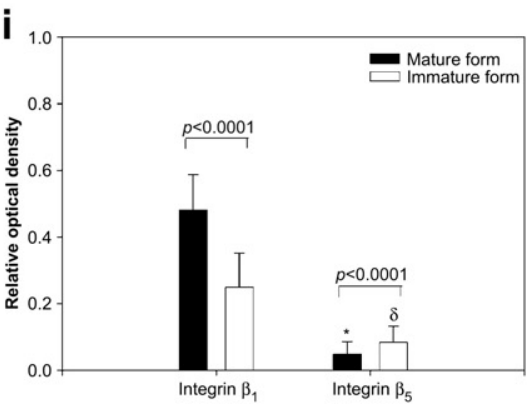

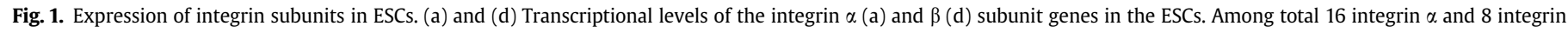

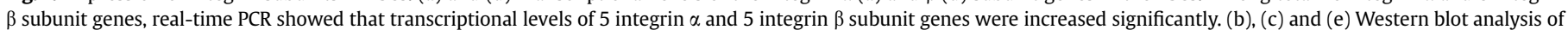

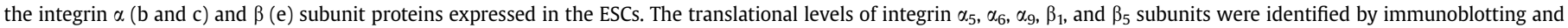

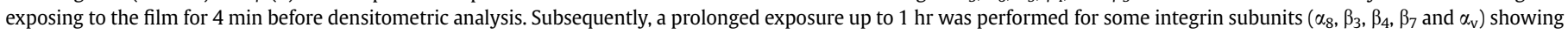

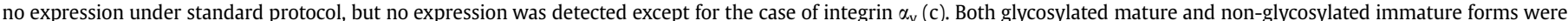

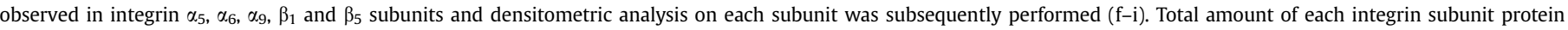

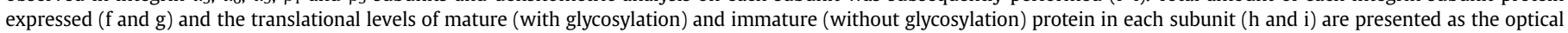

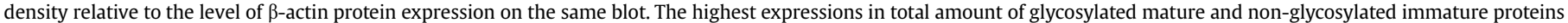

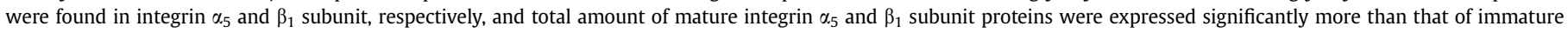

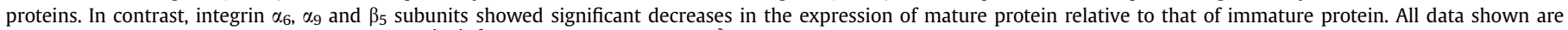
means \pm S.D. of five independent experiments. ${ }^{\text {a,b,c,d,e,f }} p<0.05,{ }^{*},{ }^{* *} p<0.05$, and ${ }^{\delta} p<0.05$. ND $=$ not detected.

$\alpha_{9}$ subunits were equal, but more translation of glycosylated mature protein was detected in integrin $\alpha_{9}$ than integrin $\alpha_{6}$ subunit (Fig. 1f,h). Integrin $\alpha_{\mathrm{v}}$ subunit showed the lowest level among all up-regulated subunits (Fig. 1c). In the case of the integrin $\beta$ subunits, only translation of $\beta_{1}$ and $\beta_{5}$ subunits was detected (Fig. 1e) and, regardless of glycosylation, the $\beta_{1}$ subunit showed more translation than the $\beta_{5}$ subunit (Fig. 1g,i). Integrin heterodimers $\alpha_{5} \beta_{1}$ and $\alpha_{6} \beta_{1}$ have been identified in ESCs to date $[26,27]$. Blocking integrin subunits $\alpha_{5}, \alpha_{6}$ and $\alpha_{\mathrm{v}}$ or heterodimer $\alpha_{9} \beta_{1}$ in ESCs resulted in lowered adhesion to fibronectin, laminin, vitronectin and tenascin-C ECM components compared with non-blocking (Supplementary Fig. 1).

\subsection{Optimization of the mechanical properties of a 3D scaffold for self-renewal of ESCS}

We sought to utilize a hydrogel system developed by our laboratory as a 3D synthetic ECM analog [23,28], here using ligation of the above-discovered integrin heterodimers for supporting selfrenewal of ESCs. A hydrogel network is formed in situ, in the presence of suspended cells, by crosslinking a reactive PEG vinylsulfone (PEG-VS) under near-physiological conditions with a di-cystyl peptide, the cysteine residues flanking a matrix metalloproteinase substrate site; adhesion ligands, formed as synthetic peptides with a free cysteine residue near the $\mathrm{N}$-terminus, are linked within the network by the same reaction (Supplementary Fig. 2a). The mechanics of the hydrogel scaffold can be modulated by adjusting the stoichiometric ratio $(r)$ of VS to cysteine equivalents. Neither a difference in mechanical elastic modulus (obtained by varying $r$ from 0.6 to 1.1 ), nor difference in culture dimension (3D PEG-hydrogel scaffold vs. 2D coculture with or without a MEF feeder) was observed to influence the metabolic activity of ESCs (Supplementary Fig. 2c,e). Spherical ESC colonies formed uniformly $5 \mathrm{~d}$ after culture within PEG-hydrogels of any $r$ level (Supplementary Fig. 2b upper panels), and the colonies were homogenously derived from the stem cells (Supplementary Fig. 2b lower panels). A 
significant increase in the size of ESC colonies was detected in PEGhydrogel of $r$ from 0.6 or 1.1, i.e. the soft materials at the extrema of the range we explored. Whereas, despite PEG-hydrogel with $1.0 r$ was softer than those with $0.6 r$, the significantly smaller size of colonies was observed (Supplementary Fig. 2d). This suggests that mechanical property of PEG-hydrogel is not the only factor that influences the proliferation of ESCs. Significant decreases in alkaline phosphatase (AP) activity and transcription of stemnessrelated genes were detected in the ESCs cultured in the absence of a MEF feeder layer. However, such decreases disappeared by the use of the 3D scaffold of different properties (Supplementary Fig. 2f,g); no prominent dependence in AP activity upon $r$ was observed. The ESCs cultured within 3D scaffolds showed higher transcription of all stemness-related genes than the ESCs cultured in the feeder-free 2D system and, except for Foxd3, lower transcription than the ESCs cultured in the feeder-containing 2D system. Among the scaffolds of different $r$, the PEG-hydrogel of $r=0.7$ fully supported ESC maintenance and was selected for further exploration.

\subsection{Determination of adhesion peptide sequence binding specificity}

We explored the effect of integrin heterodimer ligation upon self-renewal of undifferentiated ESCs. Based on the characterization described above, we selected integrins $\alpha_{5} \beta_{1}, \alpha_{\mathrm{v}} \beta_{5}, \alpha_{6} \beta_{1}$ and $\alpha_{9} \beta_{1}$ for investigation (Fig. 1), using integrin-specific peptides for $\alpha_{5} \beta_{1}$ plus $\alpha_{v} \beta_{5}, \alpha_{6} \beta_{1}$, and $\alpha_{9} \beta_{1}$. The sequence RGDSP, derived from fibronectin, was used to primarily recognize integrin $\alpha_{5} \beta_{1}[29,30]$ and $\alpha_{v} \beta_{5}$; in this context, the soluble peptide prominently inhibited binding of ESCs to fibronectin (Supplementary Fig. 3a) as well as to vitronectin [31] (Supplementary Fig. 3b). Conversely, suppressed attachment of ESCs to RGDSP peptide incorporated within the PEG hydrogels was detected after co-treatment of anti-integrin $\alpha_{5}$ and anti-integrin $\alpha_{\mathrm{v}}$ antibodies, more so than in response to either blocking antibody alone (Supplementary Fig. 3c). The angiogenesis inducer CCN1 [32]-derived sequence TTSWSQ was utilized for specificity to integrin $\alpha_{6} \beta_{1}$ activation. The transcriptional level of stemness-related genes in ESCs was prominently increased by the addition of soluble TTSWSQ peptide inside PEG-hydrogel, while anti-integrin $\alpha_{6}$ antibody treatment significantly decreased the transcriptional levels even after being exposed to TTSWSQ peptide (Supplementary Fig. 4). The tenascin-C-derived sequence AEIDGIEL [33] was used for specificity versus integrin $\alpha_{9} \beta_{1}$; this peptide when soluble significantly inhibited attachment to tenascin-C (Supplementary Fig. 3d) and treatment with anti-integrin $\alpha_{9} \beta_{1}$ antibody remarkably blocked the binding of ESCs to AEIDGIEL peptide (Supplementary Fig. 3e). From these results, we confirmed the specificity of peptides containing RGDSP, TTSWSQ and AEIDGIEL for integrin $\alpha_{5} \beta_{1}$ as well as $\alpha_{v} \beta_{5}$, integrin $\alpha_{6} \beta_{1}$, and integrin $\alpha_{9} \beta_{1}$, respectively.

\subsection{Effects of signaling through individual integrin ligation on ESC pluripotency}

Having confirmed integrin specificity, we examined whether conjugation of the PEG-hydrogel with the adhesion peptides RGDSP, TTSWSQ or AEIDGIEL individually could support ESC self-
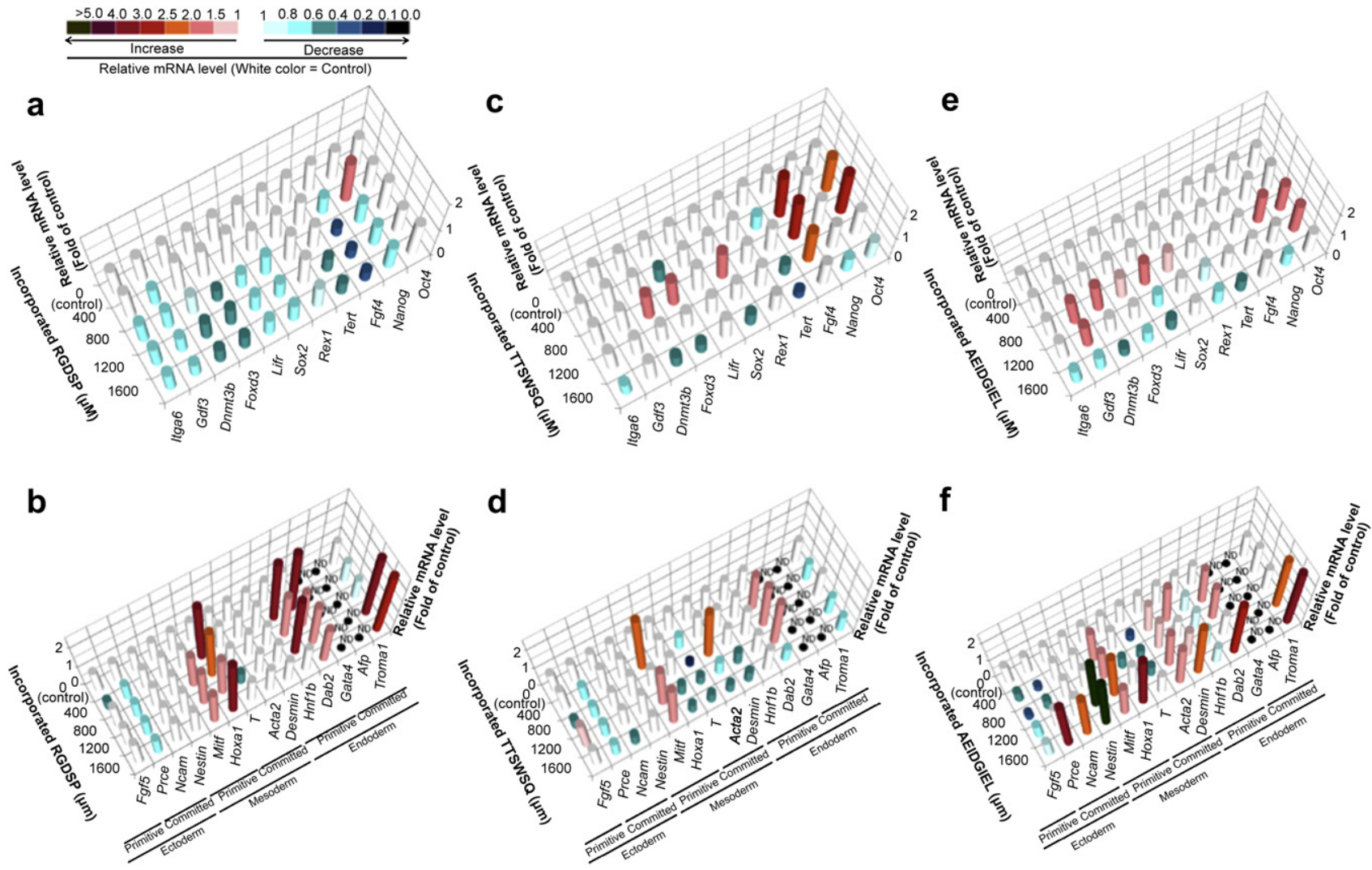

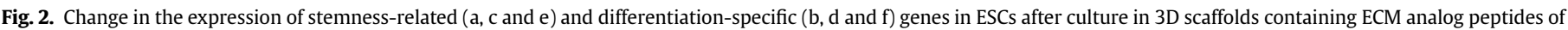

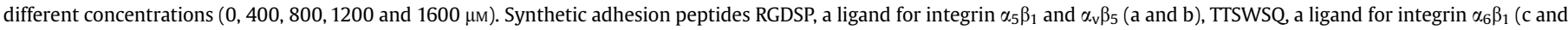

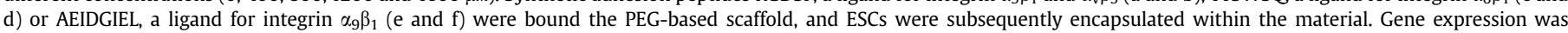

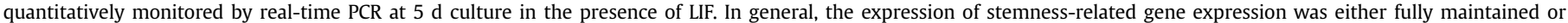

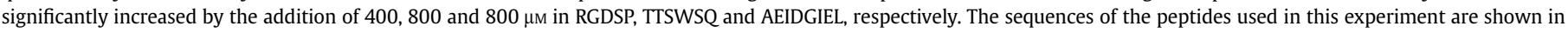
Supplementary Table 3. All data shown are means of 4 independent experiments. Different colors indicate significant difference $(p<0.05)$. 
renewal, or alternatively would lead to differentiation. Zero, 400, 800,1200 and $1600 \mu \mathrm{m}$ of each adhesion peptide were bound individually within the scaffold. Co-activation of integrin $\alpha_{5} \beta_{1}$ and $\alpha_{\mathrm{v}} \beta_{5}$ by $400 \mu \mathrm{m}$ RGDSP-containing adhesion peptide led to upregulation of only Nanog among the stemness-related genes examined (Fig. 2a), while Fgf4 and Gdf4 were down-regulated. At higher concentrations, all stemness-related genes examined were down-regulated, with the exception of Oct4, which was neither upnor down-regulated at any RGDSP concentration explored. Next, integrin $\alpha_{6} \beta_{1}$ and integrin $\alpha_{9} \beta_{1}$ were activated by TTSWSQ- and AEIDGIEL-containing hydrogels (Fig. 2c,e), respectively. Activation by $800 \mu \mathrm{m}$ TTSWSQ or AEIDGIEL strongly up-regulated the expression of most stemness-related genes (Oct4, Fgf4, Sox2, Foxd3 and Dnmt3b by integrin $\alpha_{6} \beta_{1}$ and Oct4, Nanog, Sox2, Lifr, Foxd3, Dnmt3b and Gdf3 by integrin $\alpha_{9} \beta_{1}$ ), while the same peptides of different concentrations were not suitable for the observed upregulation. No difference in transcription was detected in Gdf3, Lifr and Rex 1 by TTSWSQ-stimulated integrin $\alpha_{6} \beta_{1}$, and Fgf4 by AEIDGIEL-stimulated integrin $\alpha_{9} \beta_{1}$. On the other hand, extensive alterations were observed in the transcriptional levels of the differentiation-specific genes, while no dose-response behavior for up- or down-regulation was detected after the stimulations, other than a dramatic increase derived from the highest dose of AEIDGIEL (Fig. 2b,d,f). These results indicated that functional activation of several integrin heterodimers by adhesion peptide sequences being conjugated with PEG-hydrogel scaffold was critical for self-renewal of ESCs and that ligation of one integrin heterodimer alone could not trigger sufficient signal for self-renewal of ESCs.

\subsection{Development of synthetic 3D integrin-stimulating matrices supporting maintenance of ESC pluripotency}

To maximize the signal of integrin heterodimers for stem cell self-renewal, combinatorial conjugation of each adhesion peptide within the scaffold was conducted. Combinatorial activation of each integrin heterodimer significantly up-regulated transcription of stemness-related genes (Fig. 3a) and down-regulated transcription of differentiation-specific genes (Fig. 3b). The ESCs exposed to all three adhesion peptides for co-stimulation of $\alpha_{5} \beta_{1}, \alpha_{\mathrm{v}} \beta_{5}, \alpha_{6} \beta_{1}$ and $\alpha_{9} \beta_{1}$ increased the transcription of all stemness-related genes, which became similar to that of ESCs cultured on MEF feeder layers. In contrast, ESCs treated with blocking antibodies of integrin heterodimers on feeder layers prominently down-regulated transcription of most stemness-related genes, which was influenced by combinatorial treatment of antibodies. Co-treatment of all antibodies for the inhibition of all integrin heterodimers induced significantly reduced transcription of all the stemness-related genes, except for Tert. Thus, activation of ESCs by binding to the artificial ECM via $\alpha_{5} \beta_{1}, \alpha_{v} \beta_{5}, \alpha_{6} \beta_{1}$ and $\alpha_{9} \beta_{1}$ all together maintained ESC stemness as on MEF feeder layers, and these integrins were demonstrated to be of relevance on the feeder layers as well, shown through inhibition with blocking antibodies (Supplementary Fig. 5).

On the other hand, no significant up-regulation was detected in the genes related to differentiation except for mesoderm-specific Acta2. Compared with the ESCs cultured on a MEF feeder layer, ectoderm- and mesoderm-specific genes were down-regulated, while no significant difference was detected in endoderm-specific genes. The translation of SSEA-1 (Fig. 4a), Oct4 (Fig. 4b), and Nanog (Fig. 4c) was maintained in the ESCs cultured within the PEGhydrogel containing the RGDSP, TTSWSQ and AEIDGIEL peptides for 3 wk without subpassage. Especially, Nanog translation by hydrogel-embedded ESCs activated by integrin $\alpha_{9} \beta_{1}$ or all four integrin heterodimers prominently increased, which become similar to that of the ESCs cultured on the feeder layers. In addition, the ESCs cultured within the PEG-hydrogel conjugated with all three adhesion peptides formed spherical colonies (Supplementary Fig. 6) and significantly promoted proliferation and survival (Supplementary Fig. 7). However, this level of survival (mean \pm S.D.; $76.3 \pm 1.33 \%$ ) was significantly lower than those of ESCs cultured in a 2D microenvironment with $(84.5 \pm 6.95 \%)$ or without $(84.4 \pm 6.95 \%)$ a MEF feeder layer. Thus, these studies showed that a synthetic 3D microenvironment activating integrin $\alpha_{5} \beta_{1}$ and $\alpha_{\mathrm{v}} \beta_{5}$, integrin $\alpha_{9} \beta_{1}$
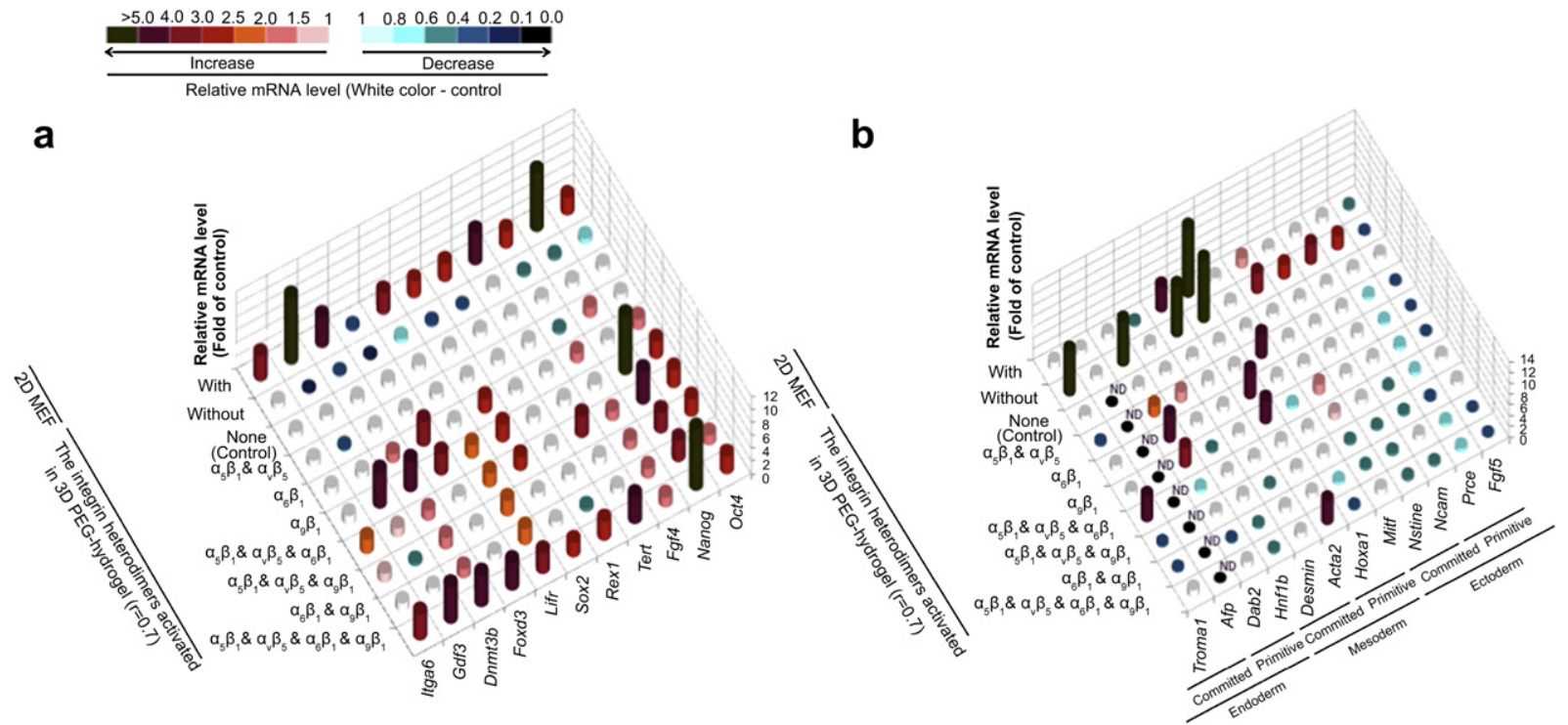

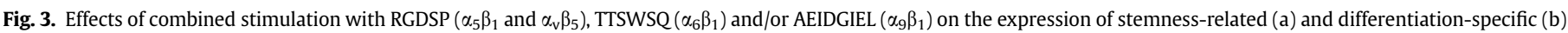

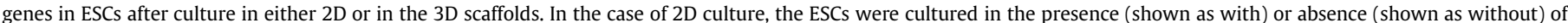

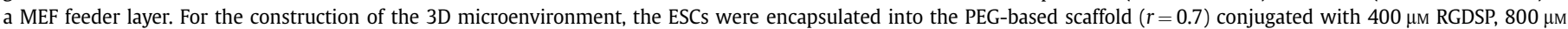

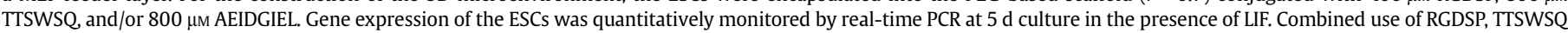

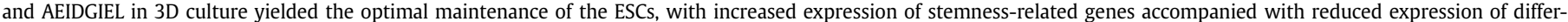

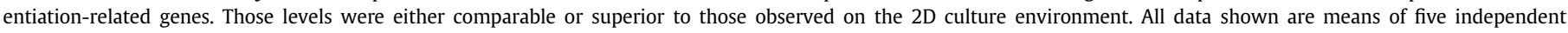
experiments. Different colors indicate significant difference $(p<0.05)$. ND $=$ not detected. 

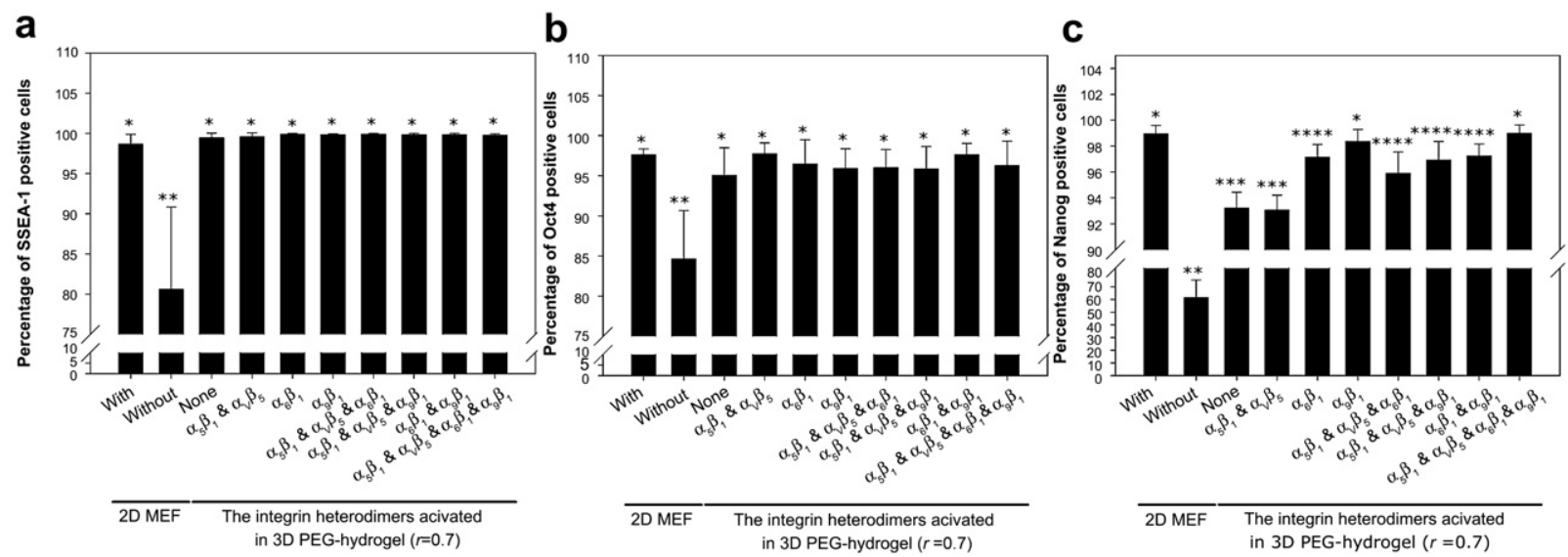

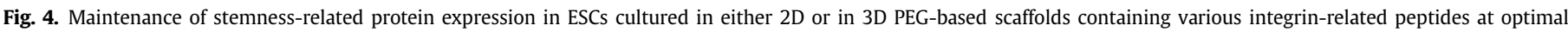

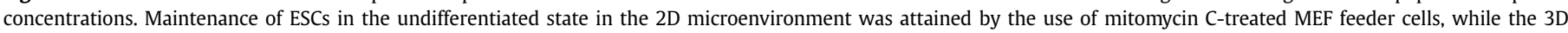

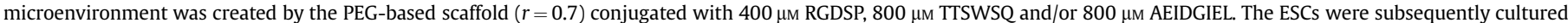

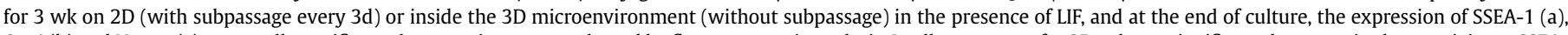

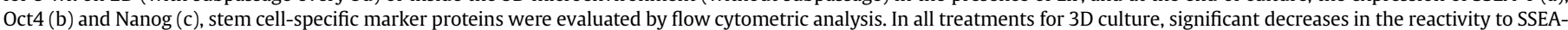

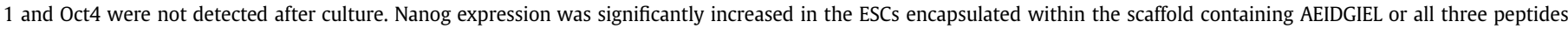

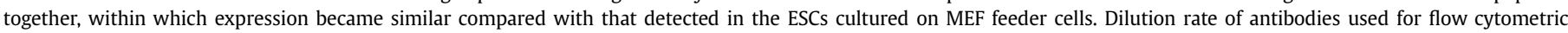
analysis are listed in Supplementary Table 2 and all data shown are means \pm S.D. of three independent experiments. ${ }^{* * * * * * * * * * *} p<0.05$.

and integrin $\alpha_{6} \beta_{1}$ could effectively support self-renewal of ESCs even without feeder cells, up-regulating stemness and downregulating differentiation-related genes.

\subsection{Regulation of stemness-related signal molecule activity by downstream signaling of integrin heterodimers within $3 D$, RGDSP, TTSWSQ AIEDGIEL-containing scaffolds}

For identifying signal proteins regulated by downstream signaling derived from combinatorial activation of each integrin heterodimer, we investigated transcription and translation of Stat3 [34,35], $\beta$-catenin [36,37], Smad 1/5/8 [38] and Akt1 [39,40] genes and proteins, which have been reported as key signal molecules supporting stem cell self-renewal. In the case of Stat 3 activated by LIF (Supplementary Fig. 8a,b,c), co-stimulation of all four integrin heterodimers stimulated Stat3 transcription, while the stimulation of integrin heterodimers within the 3D scaffold significantly down-regulated the activation of Stat3, regardless of type of adhesion peptides. In the case of $\beta$-catenin transcription factor regulated by Wnt (Supplementary Fig. 8d,e), the highest $\beta$-catenin gene and protein expression were observed in the ESCs cultured on MEF feeder layers. There were significant transcriptional up- or down-regulations in the ESCs stimulated by specific integrin heterodimers inside a 3D scaffold. However, no translational regulation was detected and merely the absence of MEF feeder cells or the 3D scaffold prominently down-regulated $\beta$-catenin translation. In the case of Smad 1/5/8 signaling derived from BMP4 (Supplementary Fig. 9), ESCs significantly changed the transcription of Smad 1, 5 and 8 in response to external stimulation of the integrin heterodimers. However, no alteration in the activation of Smad $1 / 5 / 8$ was detected by any of these stimulations. A significant increase in the transcription of the kinase Akt1, which is activated by unknown factor-stimulated phosphoinositide 3-kinase (PI3K), was detected after the stimulation of integrin $\alpha_{6} \beta_{1}$ or all integrin heterodimers inside PEG-hydrogel (Fig. 5). Even the 3D scaffold itself, without conjugation of adhesion peptide, significantly stimulated Akt1 transcription. However, there was no correlation between the regulation of Akt1 transcription and that of total Akt1 translation. Mere activation of integrin $\alpha_{6} \beta_{1}$, integrin $\alpha_{6} \beta_{1}$ and $\alpha_{9} \beta_{1}$ together, or $\alpha_{5} \beta_{1}, \alpha_{v} \beta_{5}, \alpha_{6} \beta_{1}$ and $\alpha_{9} \beta_{1}$ heterodimer signaling all together within the PEG-hydrogel significantly increased Akt1 activation. The differences in response of the ESCs between culture on MEFs and culture in the 3D matrix in terms of Smad $1 / 5 / 8$, Stat 3 and $\beta$-catenin activation indicate that different pathways of signal transduction can lead to the same overall cellular phenotype of maintenance of the undifferentiated state. Still, based on the results, we can presume that this Akt1 signal has a critical effect on stem cell self-renewal and survival within this material.

\section{Discussion}

Numerous signals must be integrated by the ESCs within the niche, to drive self-renewal versus differentiation decisions. Many of these derived from the ECM, however the roles they play, in alternately supporting self-renewal or differentiation, remains unclear. To address this, we developed an artificial ECM analog, with which we could explore the role of integrin ligation and activation on ESC fate decisions. Through transcriptional analysis of 24 integrin subunits, followed by confirmation at the protein level and inhibition with blocking antibodies, we identified $\alpha_{5} \beta_{1}, \alpha_{v} \beta_{5}$, $\alpha_{6} \beta_{1}$ and $\alpha_{9} \beta_{1}$ as interesting. Using the 3D hydrogel microenvironment as an experimental tool, we determined that activation of these four integrins together, but not alone, led to up-regulation of stemness genes and down-regulation of differentiation genes. Integrin signaling apparently plays a role in ESC interaction with feeder cells as well, in that blocking binding of these same integrins on MEF feeder layers also inhibited maintenance of stemness. Within the hydrogel that binds $\alpha_{5} \beta_{1}, \alpha_{v} \beta_{5}, \alpha_{6} \beta_{1}$ and $\alpha_{9} \beta_{1}$, Akt1derived signal transduction along with regulation of other stemness-related gene appears to be a major key for maintaining self-renewal of ESCs and inducing additional morphogenesis such as the formation of spherical colonies.

From a practical perspective, the hydrogels presented herein may be useful not just to study the roles of integrins in ESC decision making, but also as a substrate for ESC self-renewal in culture, in the absence of MEF feeder cells. We note that other approaches, both based on matrices [5,41] and molecular [36,38] approaches have been presented. The matrix approach described here is particularly flexible, and moreover may be useful in investigating 
a

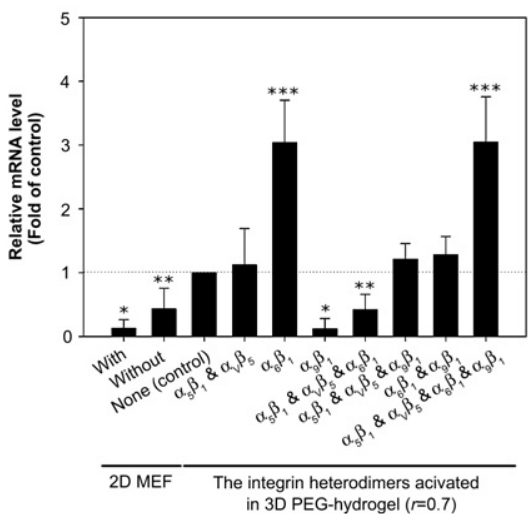

b

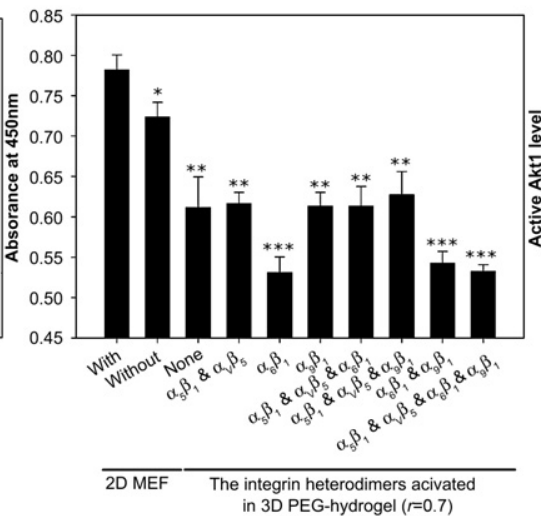

C

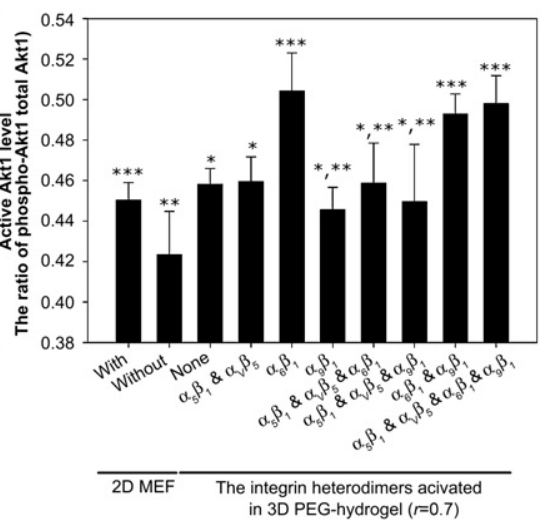

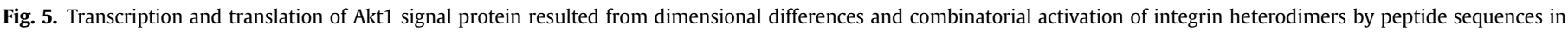

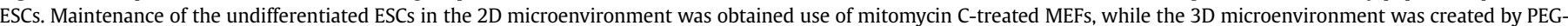

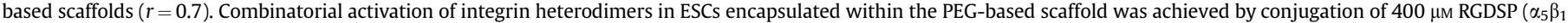

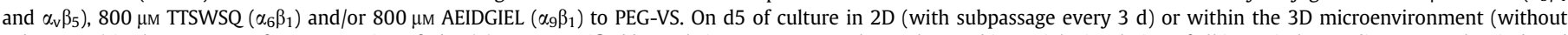

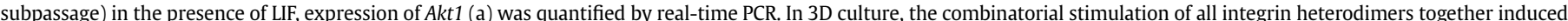

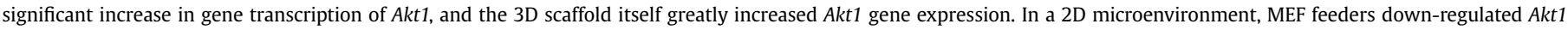

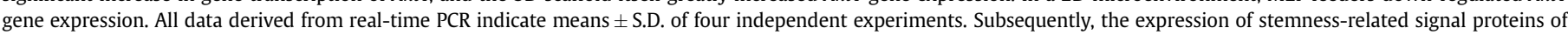

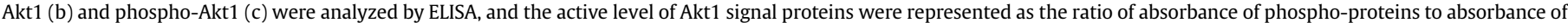

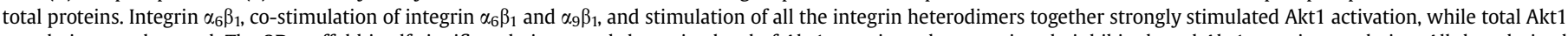

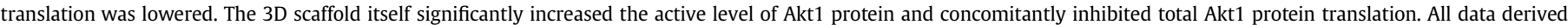
from ELISA indicate means \pm S.D. of five independent experiments. ${ }^{*}, * * * * * p<0.05$.

and applying the influence of matrix interactions in the niche upon stem cell self-renewal and differentiation more generally.

\section{Conclusions}

We present an approach by which to qualitatively and quantitatively characterize the requirements for integrin ligation in the embryonic stem cell microenvironment necessary for maintenance of stemness. The hydrogel material allows systematic modulation of mechanics, adhesion ligand(s), amounts of adhesion ligands, and proteolytic sensitivity. Although we have demonstrated the approach here with embryonic stem cells, the method is equally applicable to adult stem cells as well. From the perspective of the analysis we performed on murine embryonic stem cells, we applied the method to demonstrate a critical role of simultaneous signaling from identified $\alpha_{5} \beta_{1}, \alpha_{v} \beta_{5}, \alpha_{6} \beta_{1}$ and $\alpha_{9} \beta_{1}$ in maintenance of stemness. This combination of integrins may specific to murine embryonic stem cells, and comparative analysis in human embryonic stem cells is both necessary and under way.

\section{Acknowledgements}

The authors thank Kil Ah Kim for assistance with Western blot analysis. Partial financial support from European Commission projects Expertissues (to JAH) and Genostem (to JAH) and from the Korean Stem Cell Research Center (to JML) is gratefully acknowledged.

\section{Appendix. Supplementary data}

Supplementary data associated with this article can be found in the online version, at doi:10.1016/j.biomaterials.2009.10.054.

\section{References}

[1] Mallon BS, Park K-Y, Chen KG, Hamilton RS, McKay RDG. Toward xeno-free culture of human embryonic stem cells. Int J Biochem Cell Biol 2006;38: 1063-75.
[2] Skottman H, Hovatta O. Culture conditions for human embryonic stem cells. Reproduction 2006;132:691-8.

[3] McDevitt TC, Palecek SP. Innovation in the culture and derivation of pluripotent human stem cells. Curr Opin Biotechnol 2008;19:527-33.

[4] Li YJ, Chung EH, Rodriguez RT, Firpo MT, Kevin E. Healy. Hydrogels as artificial matrices for human embryonic stem cell self-renewal. J Biomed Mater Res A 2006;79:1-5.

[5] Nur-E-Kamal A, Ahmed I, Kamal J, Schindler M, Meiners S. Three-dimensional nanofibrillar surfaces promote self-renewal in mouse embryonic stem cells. Stem Cells 2006;24:426-33.

[6] Kloxin AM, Kasko AM, Salinas CN, Anseth KS. Photodegradable hydrogels for dynamic tuning of physical and chemical properties. Science 2009;324:59-63.

[7] Hynes RO. Integrins: bidirectional, allosteric signaling machines. Cell 2002;110:673-87.

[8] Hata RI. Where am I? How a cell recognizes its positional information druing morphogenesis. Cell Biol Int 1996;20:59-65.

[9] Boudreau N, Bissell MJ. Extracellular matrix signaling: integration of form and function in normal and malignant cells. Curr Opin Cell Biol 1998;10:640-6.

[10] Danen EH, Yamada KM. Fibronectin, integrins, and growth control. J Cell Physiol 2001;189:1-13.

[11] Ramirez F, Rifkin DB. Cell signaling events: a view from the matrix. Matrix Biol 2003;22:101-7.

[12] Cooper AR, MacQueen HA. Subunits of laminin are differentially synthesized in mouse eggs and early embryos. Dev Biol 1983;96:467-71.

[13] George EL, Georges-Labouesse EN, Patel-King RS, Rayburn H, Hynes RO. Defects in mesoderm, neural tube and vascular development in mouse embryos lacking fibronectin. Development 1993;119:1079-91.

[14] Fässler R, Meyer M. Consequences of lack of beta 1 integrin gene expression in mice. Genes Dev 1995;9:1896-908.

[15] Stephens LE, Sutherland AE, Klimanskaya IV, Andrieux A, Meneses J, Pedersen RA, et al. Deletion of beta 1 integrins in mice results in inner cell mass failure and peri-implantation lethality. Genes Dev 1995;9:1883-95.

[16] Li S, Harrison D, Carbonetto S, Fassler R, Smyth N, Edgar D, et al. Matrix assembly, regulation, and survival functions of laminin and its receptors in embryonic stem cell differentiation. J Cell Biol 2002;157:1279-90.

[17] Watt FM, Hogan BL. Out of eden: stem cells and their niches. Science 2000;287:1427-30.

[18] Czyz J, Wobus A. Embryonic stem cell differentiation: the role of extracellular factors. Differentiation 2001;68:167-74.

[19] Williams DA, Rios M, Stephens C, Patel VP. Fibronectin and VLA-4 in haematopoietic stem cell-microenvironment interactions. Nature 1991;352: 438-41.

[20] Zhu AJ, Haase I, Watt FM. Signaling via beta1 integrins and mitogen-activated protein kinase determines human epidermal stem cell fate in vitro. Proc Nat Acad Sci USA 1999;96:6728-33.

[21] Campos LS, Leone DP, Relvas JB, Brakebusch C, Fassler R, Suter U, ffrenchConstant C. Beta1 integrins activate a MAPK signalling pathway in neural stem cells that contributes to their maintenance. Development 2004; $131: 3433-44$. 
[22] Lutolf MP, Hubbell JA. Synthetic biomaterials as instructive extracellular microenvironments for morphogenesis in tissue engineering. Nat Biotechnol 2005;23:47-55.

[23] Lutolf MP, Weber FE, Schmoekel HG, Schense JC, Kohler T, Muller R, et al. Repair of bone defects using synthetic mimetics of collagenous extracellular matrices. Nat Biotechnol 2003;21:513-8.

[24] Lutolf MP, Tirelli N, Cerritelli S, Cavalli L, Hubbell JA. Systematic modulation of Michael-type reactivity of thiols through the use of charged amino acids. Bioconj Chem 2001;12:1051-6.

[25] Lutolf MP, Hubbell JA. Synthesis and physicochemical characterization of endlinked poly(ethylene glycol)-co-peptide hydrogels formed by Michael-type addition. Biomacromolecules 2003;4:713-22.

[26] Cooper HM, Tamura RN, Quaranta V. The major laminin receptor of mouse embryonic stem cells is a novel isoform of the alpha 6 beta 1 integrin. J Cell Biol 1991;115:843-50.

[27] Fassler R, Pfaff M, Murphy J, Noegel A, Johansson S, Timpl R, et al. Lack of beta 1 integrin gene in embryonic stem cells affects morphology, adhesion, and migration but not integration into the inner cell mass of blastocysts. J Cell Biol 1995;128:979-88.

[28] Raeber GP, Lutolf MP, Hubbell JA. Molecularly engineered PEG hydrogels: a novel model system for proteolytically mediated cell migration. Biophys J 2005;89:1374-88.

[29] Pierschbacher MD, Ruoslahti E. Cell attachment activity of fibronectin can be duplicated by small synthetic fragments of the molecule. Nature 1984;309:30-3.

[30] Mardilovich A, Kokkoli E. Biomimetic peptide-amphiphiles for functional biomaterials: the role of GRGDSP and PHSRN. Biomacromolecules 2004;5:950-7.

[31] Maubant S, Saint-Dizier D, Boutillon M, Perron-Sierra F, Casara PJ, Hickman JA, et al. Blockade of $\alpha \mathrm{v} \beta 3$ and $\alpha \mathrm{v} \beta 5$ integrins by RGD mimetics induces anoikis and not integrin-mediated death in human endothelial cells. Blood 2006; $108: 3035-44$.
[32] Leu SJ, Liu Y, Chen N, Chen C-C, Lam SC-T, Lau LF. Identification of a nove integrin $\alpha 6 \beta 1$ binding site in the angiogenic inducer CCN1 (CYR61). J Bio Chem 2003;278:33801-8.

[33] Yokosaki Y, Matsuura N, Higashiyama S, Murakami I, Obara M, Yamakido M, et al. Identification of the ligand binding site for the integrin alpha 9 beta 1 in the third fibronectin type III repeat of tenascin-C. J Biol Chem 1998;273:11423-8.

[34] Niwa H, Burdon T, Chambers I, Smith A. Self-renewal of pluripotent embryonic stem cells is mediated via activation of STAT3. Genes Dev 1998;12:2048-60.

[35] Matsuda T, Nakamura T, Nakao K, Arai T, Katsuki M, Heike T, et al. STAT3 activation is sufficient to maintain an undifferentiated state of mouse embryonic stem cells. EMBO J 1999;18:4261-9.

[36] Sato N, Meijer L, Skaltsounis L, Greengard P, Brivanlou AH. Maintenance of pluripotency in human and mouse embryonic stem cells through activation of Wnt signaling by a pharmacological GSK-3-specific inhibitor. Nat Med 2004;10:55-63.

[37] Miyabayashi T, Teo J-L, Yamamoto M, McMillan M, Nguyen C, Kahn M. Wnt/ $\beta$ catenin/CBP signaling maintains long-term murine embryonic stem cell pluripotency. Proc Nat Acad Sci USA 2007; 104:5668-73.

[38] Ying QL, Nichols J, Chambers I, Smith A. BMP induction of Id proteins suppresses differentiation and sustains embryonic stem cell self-renewal in collaboration with STAT3. Cell 2003;115:281-92.

[39] Watanabe S, Umehara H, Murayama K, Okabe M, Kimura T, Nakano T. Activation of Akt signaling is sufficient to maintain pluripotency in mouse and primate embryonic stem cells. Oncogene 2006;25:2697-707.

[40] Hayashi Y, Furue MK, Okamoto T, Ohnuma K, Myoishi Y, Fukuhara Y, et al. Integrins regulate mouse embryonic stem cell self-renewal. Stem Cells 2007;25:3005-15

[41] Greenlee AR, Kronenwetter-Koepel TA, Kaiser SJ, Liu K. Comparison of Matrigel $^{\mathrm{TM}}$ and gelatin substrata for feeder-free culture of undifferentiated mouse embryonic stem cells for toxicity testing. Toxicol In Vitro 2005;19: 389-97. 